\title{
Correlations Between the Adenotonsillar Microbiome and Clinical Characteristics of Pediatric Patients With Snoring
}

\author{
Kyung Soo Kim (iD $\cdot$ Hyun Jin Min (i) \\ Department of Otorhinolaryngology-Head and Neck Surgery, Chung-Ang University College of Medicine, Seoul, Korea
}

Objectives. Few studies have reported combined analyses of the microbiome of the adenoids and tonsils in pediatric patients with snoring, and correlations of the adenotonsillar microbiome with clinical characteristics have not been evaluated to date. The aim of this study was to characterize the adenotonsillar microbiome and to determine its correlations with the subjective symptoms of pediatric patients with snoring and with levels of regional mucosal immune molecules.

Methods. Twenty-four children who underwent tonsillectomy with adenoidectomy owing to snoring were enrolled in this cross-sectional study conducted between August 2017 and December 2018. The microbiome of the adenoids and tonsils was characterized, and its alpha- and beta-diversity was determined. Clinical characteristics, including subjective discomfort during sleep (assessed using the obstructive sleep apnea-18 questionnaire), the presence of allergic rhinitis, concentrations of heat shock protein (Hsp)27, Hsp70, and interleukin-8 (IL-8) in lavage fluids, and white blood cell (WBC) counts, were measured.

Results. At the phylum level, the microbiome was not significantly different between the adenoids and tonsils; the alpha and beta indices were likewise not significantly different between these two regions. The alpha-diversity of the entire adenotonsillar microbiome was associated with sex, emotional stress, and IL-8 levels in the tonsil lavage fluids. Beta-diversity was associated with Hsp27 levels in the tonsil lavage fluids and WBC counts. Multiple allergen simultaneous test results were not significant, although total serum immunoglobulin E levels were significantly associated with the beta-diversity of the adenotonsillar microbiome.

Conclusion. The data reported herein suggest, for the first time, that the adenotonsillar microbiome interacts with the regional mucosal immune system. The observed association of the microbiome with subjective discomfort is a novel finding that warrants further investigation.

Keywords. Adenoid; Snoring; Tonsil; Microbiome

\section{INTRODUCTION}

Pathogenic hyperplasia of the tonsils and adenoids is one of the most frequently encountered diseases in the pediatric popula-

\footnotetext{
- Received July 31, 2020

Revised November 10, 2020

Accepted November 10, 2020

- Corresponding author: Hyun Jin Min

Department of Otorhinolaryngology-Head and Neck Surgery, Chung-Ang

University Hospital, Chung-Ang University College of Medicine,

102 Heukseok-ro, Dongjak-gu, Seoul 06973, Korea

Tel: +82-2-6299-1765, Fax: +82-2-825-1765

E-mail: jjinient@gmail.com
}

tion. The incidence of adenoidectomy has been reported to be 740 per 100,000 in children under the age of 10 years, while that of tonsillectomy is 129.4 per 100,000 [1,2]. Hyperplasia of the tonsils and adenoids is known to be associated with infectious diseases, such as recurrent tonsillitis and otitis media with effusion [3,4]. Recently, several culture-independent molecular assays based on 16S rRNA gene sequencing have shown complex, diverse, and highly variable bacterial communities to be present in the tonsil and adenoid tissues.

Adenotonsillar hypertrophy is an independent risk factor for snoring in children [5]. Although there is no definitive consensus regarding the mechanisms of adenotonsillar hypertrophy in

Copyright (C) 2021 by Korean Society of Otorhinolaryngology-Head and Neck Surgery.

This is an open-access article distributed under the terms of the Creative Commons Attribution Non-Commercial License (https://creativecommons.org/licenses/by-nc/4.0)

which permits unrestricted non-commercial use, distribution, and reproduction in any medium, provided the original work is properly cited. 
snoring and obstructive sleep apnea (OSA), the immunological mechanisms of adenotonsillar hypertrophy in OSA are suspected to be different from those in recurrent infection-associated conditions, such as tonsillitis. The local immune responses of lymphocytes have been reported to be remarkably different in patients with recurrent tonsillitis than in those with OSA [6,7]. In tonsils from patients with recurrent tonsillitis, large micro-colonies of bacteria have been observed, and large numbers of B and $\mathrm{T}$ lymphocytes have been reported immediately adjacent to the tonsil crypt itself. In contrast, no bacteria or significant skewing of the distribution of lymphocytes based on the site (such as the follicles or crypts) was observed in tonsils from patients with OSA [6]. Therefore, it is reasonable to suspect that the microbiome of the tonsil and adenoid tissues may be different between patients with infective hyperplasia and those with OSA. However, to date, studies on the tonsil and adenoid microbiomes have mainly described associations with recurrent tonsillitis or adenoids related to middle ear infection.

The palatine tonsils (generalized as tonsils here), lingual tonsils, and adenoids comprise the Waldeyer's lymphatic ring, a wellknown origin of recurrent upper respiratory tract infections during infancy. Although the tonsils and adenoids share structural similarities and are anatomically close to each other, they have different characteristics. The tonsils are located within the oropharyngeal cavity, while the adenoids are connected to the nasopharyngeal airway. The tonsils are covered with stratified squamous epithelium, with the crypts arranged in a lacunar manner; in contrast, the adenoids are covered with respiratory epithelium, with the crypts located in longitudinal folds [8]. Unlike adenoidectomy, tonsillectomy has no therapeutic effect on middle ear effusion [9].Therefore, the tonsil and adenoid microbiomes might differ.

In our previous study, we found several damage-associated molecular patterns (host molecules involved in innate immunity) expressed in tissues and present in the lavage fluids (LFs) of pediatric adenoids [10]. In this study, first, we compared the microbiome of tonsils and adenoids in pediatric patients with snoring not associated with recurrent infections. Next, we compared features of the microbiome with clinical characteristics, including subjective discomfort during sleep. Finally, we evaluated the correlations of the adenotonsillar microbiome with regional concentrations of innate immunity-associated molecules.

\section{H I G H L L I G G H T S}

- Association between microbiome profiles and clinical characteristics of pediatrics with snoring was evaluated.

- Association between microbiome profiles and mucosal immune molecules was investigated.

- We suggest that adenotonsillar microbiome interacts with the regional mucosal immune system and subjective discomfort of pediatrics with snoring.

\section{MATERIALS AND METHODS}

\section{Study design and sample collection}

This study was approved by the Institutional Review Board of the Chung-Ang University Hospital, and written informed consent was obtained from the caregivers of all participants. The study was cross-sectional, comprising of 24 pediatric patients who underwent tonsillectomy with adenoidectomy. Patients with snoring or symptoms of sleep apnea were enrolled in this study, and those with recurrent tonsillitis or antibiotic medication history within the previous 4 weeks were excluded. Further, patients with underlying conditions, such as immunodeficiency, malignancy, or allergic asthma, were excluded from the study. Medical records, including results of the multiple allergen simultaneous test (MAST; LG Life Sciences, Seoul, Korea) [11,12], total serum immunoglobulin E (IgE) levels (group 1, negative; group 2 , low titer ( $<2$ positivity; $\leq 0.70 \mathrm{IU} / \mathrm{mL}$ ); and group 3, high titer ( $\geq 2$ positivity; $0.70 \mathrm{IU} / \mathrm{mL}$ ) [13], and white blood cell (WBC) counts, and the OSA-18 questionnaire were reviewed.

The OSA-18 questionnaire assessed the quality of life, and disordered breathing and discomfort in pediatric sleep; it comprised of the following five domains: sleep disturbances, physical suffering, emotional distress, daytime problems, and parent or caretaker concern [14]. Blood tests and OSA-18 questionnaire were conducted a day before the surgery. Tonsillectomy with adenoidectomy was performed under general anesthesia by an otorhinolaryngologist, and LFs from the tonsils and adenoids (collected separately from each tissue), as well as the tonsil and adenoid tissues, including the surface mucosa and submucosal lymphoid tissues, were obtained as described previously [10]. Approximately $5 \mathrm{~mL}$ of regular saline was instilled onto the tonsil (under direct visualization) and adenoid (using a $30^{\circ}$ endoscope) surfaces, and approximately $4 \mathrm{~mL}$ retrieved after $3 \mathrm{sec}-$ onds. Next, the tonsil and adenoid tissues, including the surface mucosa and submucosal lymphoid tissues, were sampled using cutting forceps before surgery. The tissue samples were placed in a sterile container, stored on ice, and transferred to the laboratory within 1 hour. All samples were stored at $-20^{\circ} \mathrm{C}$ until further analysis.

\section{DNA extraction, 16S rRNA gene amplification, and pyrosequencing}

Total genomic DNA was extracted as described previously [15]. Microbial genomic DNA was extracted from the tonsil and adenoid biopsy specimens using the repeated-bead-beating method [16]. The barcoded forward primer 27F (5'-XXXXXXXXXXGAGTTTGATCMTGGCTCAG-3', where " $X$ " represents the barcode sequence) and reverse primer 338R (5'-TGCTGCCTCCCGTAGGAGT-3'), both ligated to an FLX Titanium adapter, were used for amplifying theV1-V2 region of the 16S rRNA gene. Diluted DNA template (8 $\mu \mathrm{L}, 1: 7)$, primer mix $(2 \mu \mathrm{L}, 0.2 \mu \mathrm{M})$, 
and $2 \times$ Ex Taq polymerase premix (TaKaRa Bio, Shiga, Japan) were mixed for the polymerase chain reaction (PCR; total reaction volume, $50 \mu \mathrm{L}$ ). PCR conditions were set as previously described [13]. QIAquick PCR purification kit (Qiagen, Hilden, Germany) was used to purify amplicons, prepared as eight replicates. Quant-iT PicoGreen double-stranded DNA kit (Invitrogen, Carlsbad, CA, USA) was used to determine the concentration of DNA. Equimolar concentrations of the prepared amplicons for 48 tonsil and adenoid samples were subjected to pyrosequencing on the 454 GS-FLX Titanium sequencing platform (Roche Diagnostics, Manheim, Germany).

\section{Analysis of microbiota in the tonsil and adenoid tissue samples}

Analysis of tissue microbiota was performed as described previously [13]. Briefly, the reads were analyzed on the Quantitative Insights Into Microbial Ecology (QIIME) platform [16], and operational taxonomic units (OTUs) ( $\geq 97 \%$ identity) were selected using the Greengenes database [17]. After excluding unassigned or non-bacterial kingdom-assigned sequences and singletons, $6,015,107$ reads were obtained $(125,314 \pm 28,769$ reads per sample). Alpha-diversity was measured using Shannon diversity index, Chao1, and observed OTUs. Beta-diversity was evaluated using principal component analysis ( $\mathrm{PCoA}$ ) based on weighted and unweighted UniFrac and Bray-Curtis distances.

\section{Measurement of heat shock protein and interleukin-8 levels in the tonsil and adenoid LFs}

The amounts of extracellular heat shock protein (Hsp)27, Hsp70, and interleukin-8 (IL-8) in the LFs were determined using a commercial enzyme-linked immunosorbent assay kit (R\&D Systems, Minneapolis, MN, USA), according to the manufacturer's instructions.

\section{Statistical analysis}

Mann-Whitney U-test and Spearman's rank correlation were performed using GraphPad Prism software ver. 5.0 (GraphPad, San Diego, CA, USA). Linear discriminant analysis (LDA) coupled with an effect size measurement (LEfSe) was employed to distinguish the taxonomic characteristics between groups [18]. Alpha-diversity (within-sample diversity; Shannon diversity index, Chao1, and observed OTUs) was calculated for each sample in the QIIME pipeline. Beta-diversity (between-sample diversity) was calculated using the phylogenetic metrics (UniFrac) for microbial community and non-phylogenetic metrics (BrayCurtis) for microbial gene community in the QIIME pipeline. The calculated distance matrices were used to perform PCoA. The analysis of similarities (ANOSIM) method was used to predict the differences in microbial communities between groups, whereas permutational multivariate analysis of variance (PERMANOVA) was used to evaluate the dissimilarities based on site (adenoid and tonsil), sex, MAST, and total serum IgE levels.

\section{RESULTS}

\section{Patient demographics}

Twenty-four patients were enrolled in this study (mean age, 6.29 years; standard deviation [SD], 6.29 years; age range, 4-12 years). Sixteen were boys and eight were girls. Nine (37.5\%) had allergic rhinitis (AR). Among the OSA-18 questionnaire domains, sleep disturbance, physical suffering, and caregiver concerns were the highest-rated, followed by daytime problems and emotional distress. The mean \pm SD levels of IL-8, Hsp27, and Hsp70 detected in the adenoid LFs were $216.97 \pm 77.61,328.39 \pm 222.47$, and $4,023.94 \pm 2,708.82 \mathrm{pg} / \mathrm{mL}$, respectively, whereas those detected in the tonsil LFs were $189.68 \pm 80.07,438.77 \pm 204.17$, and $4,756.17 \pm 2,747.32 \mathrm{pg} / \mathrm{mL}$, respectively (Table 1 ).

\section{Bacterial community composition in the tonsils and adenoids}

Tissue samples from the tonsils revealed a predominance of Proteobacteria (mostly the genus Haemophilus), Firmicutes (dominated by the genus Streptococcus), and Bacteroidetes (dominated by the genus Prevotella). Tissue samples from the adenoids were dominated by Proteobacteria (mostly the genus Haemophilus), Firmicutes (dominated by the genus Streptococcus), and Fusobacteria (dominated by the genus Fusobacterium). At the genus level, Streptococcus was abundant, while Neisseria was present to a lesser extent in the adenoids than in the tonsils (Fig. $1 \mathrm{~A}$ and $\mathrm{B})$. LEfSe was performed to obtain the cladogram representation and to identify the predominant bacteria in tonsils and adenoids. We found Neisseria to be more abundant in the tonsils than in the adenoids (Fig. 1C).

Table 1. Clinical characteristics of the patients enrolled in the study

\begin{tabular}{lc}
\hline Variable & Value \\
\hline Number & 24 \\
Sex (male:female) & $16: 8$ \\
Age (yr) & $6.29 \pm 2.34(4-12)$ \\
Body mass index (kg/m²) & $17.52 \pm 3.05$ \\
Positivity of MAST (\%) & $9(37.5)$ \\
OSA-18 (sleep disturbance) & $13.08 \pm 4.48$ \\
OSA-18 (physical suffering) & $14.79 \pm 4.65$ \\
OSA-18 (emotional stress) & $9.58 \pm 4.65$ \\
OSA-18 (daytime problems) & $9.62 \pm 4.19$ \\
OSA-18 (caregiver concern) & $13.00 \pm 5.23$ \\
Adenoid IL-8 (pg/mL) & $216.97 \pm 77.61$ \\
Adenoid Hsp27 (pg/mL) & $328.39 \pm 222.47$ \\
Adenoid Hsp70 $(\mathrm{pg} / \mathrm{mL})$ & $4,023.94 \pm 2,708.82$ \\
Tonsil IL-8 (pg/mL) & $189.68 \pm 80.07$ \\
Tonsil Hsp27 $(\mathrm{pg} / \mathrm{mL})$ & $438.77 \pm 204.17$ \\
Tonsil Hsp70 $(\mathrm{pg} / \mathrm{mL})$ & $4,756.17 \pm 2,747.32$ \\
White blood cells $\left(10^{9} / \mathrm{L}\right)$ & $7,752.08 \pm 2,272.84$ \\
Eosinophil count $\left(10^{9} / \mathrm{L}\right)$ & $226.05 \pm 121.85$ \\
\hline
\end{tabular}

Values are presented as mean \pm SD (range), mean \pm SD, or number (\%). MAST, multiple allergen simultaneous test; OSA, obstructive sleep apnea; IL-8, interleukin-8; Hsp, heat shock protein; SD, standard deviation. 

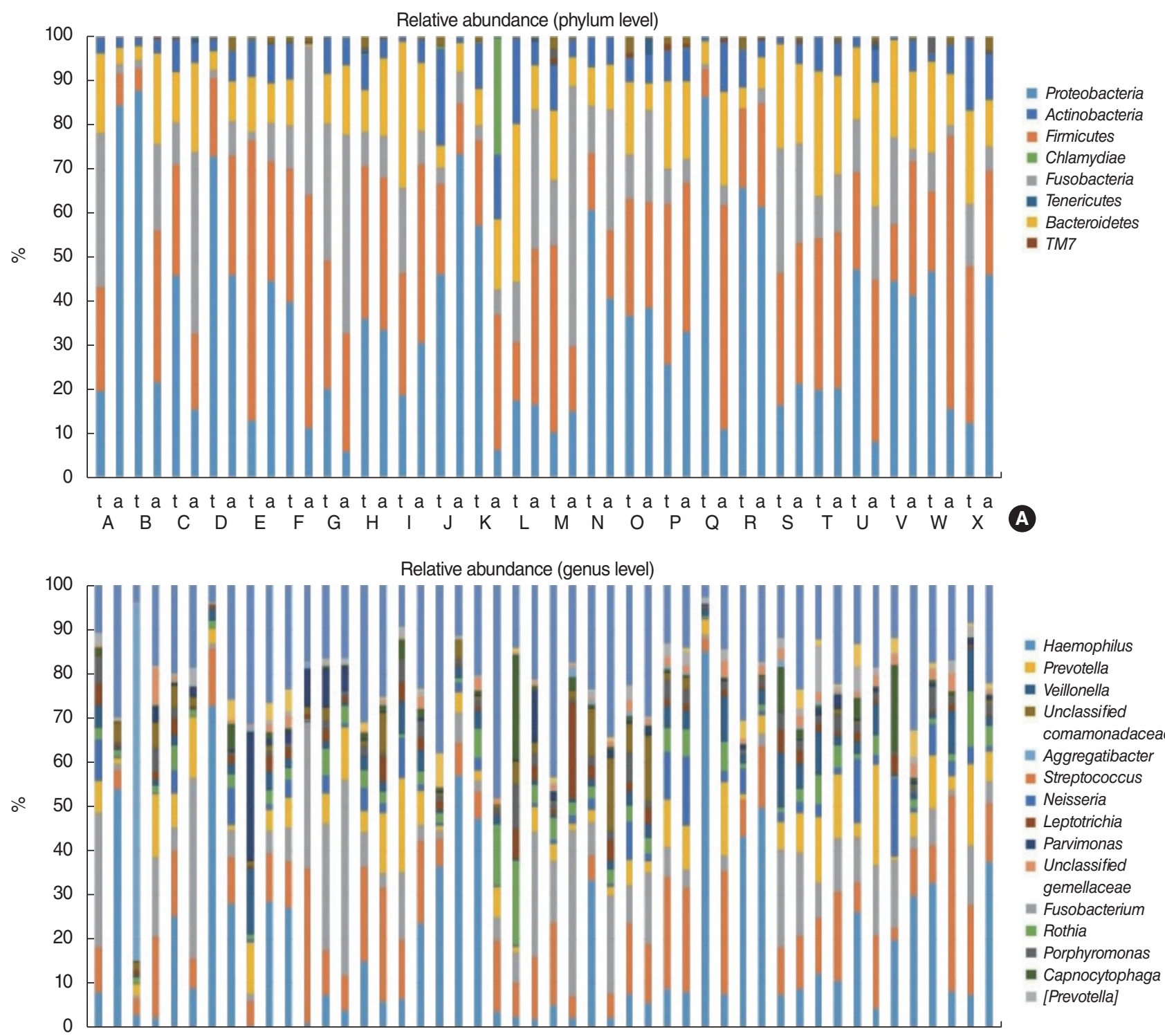

- Haemophilus

= Prevotella

- Veillonella

- Unclassified comamonadaceae

= Aggregatibacter

- Streptococcus

- Neisseria

- Leptotrichia

- Parvimonas

- Unclassified

gemellaceae

= Fusobacterium

n Rothia

- Porphyromonas

- Capnocytophaga

= [Prevotella]

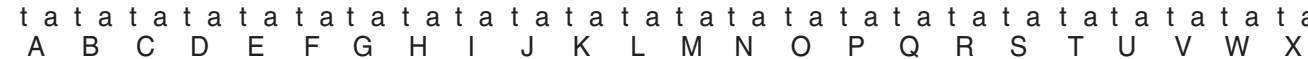

B

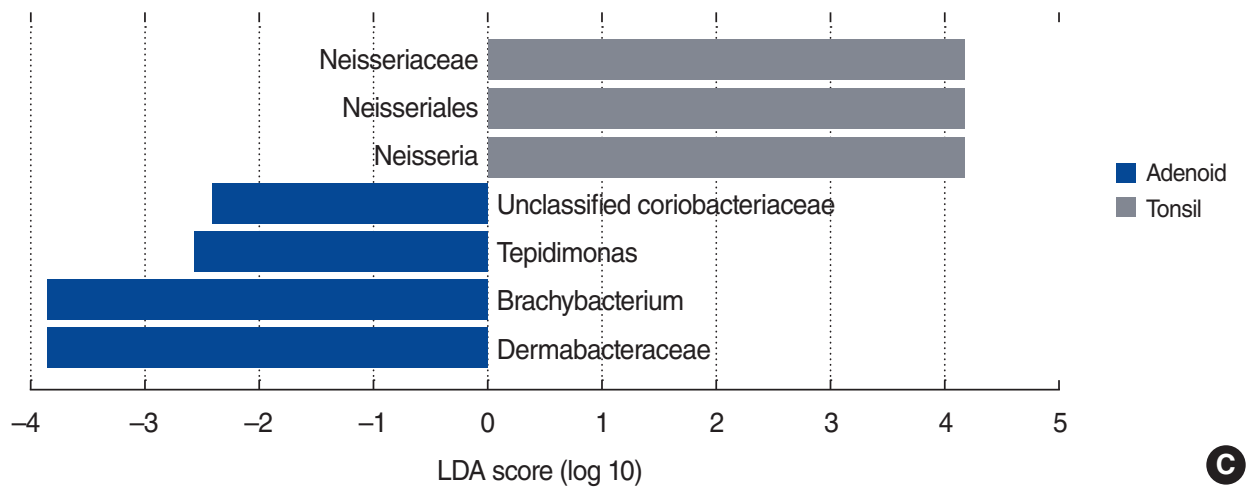

Fig. 1. Bacterial community composition of the tonsils and adenoids of 24 patients at the phylum level (A) and genus level (B). The dominant phyla and genera within the samples are shown. (C) Linear discriminant analysis (LDA) score. Histogram of the LDA scores for genera that were differentially abundant between the tonsils and adenoids. Negative (blue bars) and positive (gray bars) LDA scores represent bacterial groups in the adenoids and tonsils, respectively. 

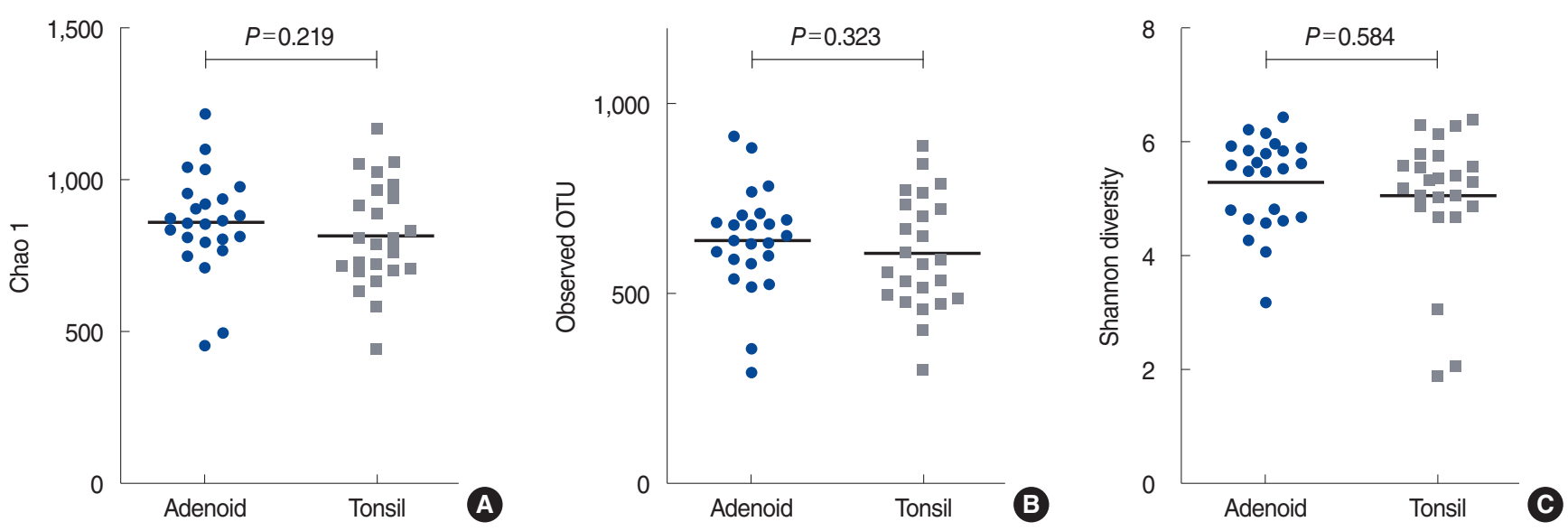

Fig. 2. Alpha-diversity in the adenoid and tonsil samples. No significant difference was observed between the adenoid and tonsil samples based on the Chao 1 (A), observed operational taxonomic units (OTUs) (B), and Shannon diversity index (C) measures.

\section{Alpha-diversity of the adenotonsillar microbiome and its correlations with clinical characteristics}

Chao1, observed OTUs, and the Shannon diversity index were used to compare the alpha-diversity of the microbiome between the adenoids and tonsils. Neither bacterial community richness nor diversity was significantly different between the adenoid and tonsil microbiomes $(P=0.219$ for Chao1, $P=0.323$ for observed OTUs, and $P=0.584$ for Shannon diversity) (Fig. 2). We evaluated the correlations of the alpha-diversity of the entire adenotonsillar microbiome with clinical characteristics. Bacterial diversity, as represented by Shannon diversity, differed significantly between males and females; males had a more diverse adenotonsillar microbiome than females $(P=0.045)$. The score of the emotional stress domain in the OSA-18 questionnaire $(P=0.047$, rho $=-0.287$ for Chao1; $P=0.014$, rho $=-0.350$ for observed OTUs) and the IL- 8 concentration in the tonsil LFs $(P=0.028$, rho $=-0.310$ for Chao1; $P=0.001$, rho $=-0.438$ for observed OTUs) were negatively correlated with the alpha-diversity of the adenotonsillar microbiome. This suggested that higher levels of emotional stress in OSA or IL-8 concentrations in the tonsil LFs were associated with lower richness of the adenotonsillar bacterial community (Table 2). However, the alphadiversity of the adenotonsillar microbiome based on MAST positivity or total serum IgE levels revealed no significant difference in Chao1, observed OTUs, and Shannon diversity (Supplementary Fig. 1).

\section{Beta-diversity of the adenotonsillar microbiome and its correlations with clinical characteristics}

Since the UniFrac distance considers the phylogenetic relatedness of OTUs with the Bray-Curtis distance, which quantifies the compositional dissimilarity between two different sites based on the counts at each site, it is only a measure of abundance [19]. We generated PCoA plots for the Bray-Curtis, unweighted UniFrac, and weighted UniFrac distances of the phylogenetic tree.
Table 2. Correlations between alpha-diversity indices and clinical characteristics

\begin{tabular}{|c|c|c|c|}
\hline \multirow[b]{2}{*}{ Characteristics } & \multicolumn{3}{|c|}{$P$-value } \\
\hline & Chao1 & $\begin{array}{l}\text { Observed } \\
\text { OTUs }\end{array}$ & $\begin{array}{l}\text { Shannon } \\
\text { diversity }\end{array}$ \\
\hline Sex & 0.991 & 0.947 & 0.045 \\
\hline Age & 0.655 & 0.810 & 0.495 \\
\hline Positivity of MAST & 0.529 & 0.496 & 0.475 \\
\hline OSA-18 (sleep disturbance) & 0.903 & 0.869 & 0.438 \\
\hline OSA-18 (physical suffering) & 0.636 & 0.319 & 0.264 \\
\hline OSA-18 (emotional stress) & $\begin{array}{c}0.047 \\
(\mathrm{rho}=-0.287)\end{array}$ & $\begin{array}{c}0.014 \\
(\mathrm{rho}=-0.350)\end{array}$ & $\begin{array}{l}0.588 \\
0.583\end{array}$ \\
\hline OSA-18 (daytime problems) & 0.217 & $\begin{array}{c}0.030 \\
(\mathrm{rho}=-0.310)\end{array}$ & 0.548 \\
\hline OSA-18 (caregiver concern) & 0.448 & 0.164 & 0.844 \\
\hline Adenoid IL-8 (pg/mL) & 0.322 & 0.206 & 0.070 \\
\hline Adenoid Hsp27 (pg/mL) & 0.079 & 0.220 & 0.115 \\
\hline Adenoid Hsp70 (pg/mL) & 0.536 & 0.421 & 0.862 \\
\hline Tonsil IL-8 (pg/mL) & $\begin{array}{c}0.028 \\
(r h o=-0.310)\end{array}$ & $\begin{array}{c}0.001 \\
(\mathrm{rho}=-0.438)\end{array}$ & 0.129 \\
\hline Tonsil Hsp27 (pg/mL) & 0.218 & 0.568 & 0.396 \\
\hline Tonsil Hsp70 (pg/mL) & 0.512 & 0.294 & 0.352 \\
\hline White blood cells $\left(10^{9} / L\right)$ & 0.053 & 0.063 & 0.745 \\
\hline Eosinophil count $\left(10^{9} / \mathrm{L}\right)$ & 0.988 & 0.602 & 0.300 \\
\hline
\end{tabular}

OTU, operational taxonomic unit; MAST, multiple allergen simultaneous test; OSA, obstructive sleep apnea; IL-8, interleukin-8; Hsp, heat shock protein.

PCoA, which showed a dissimilarity between the adenoids and tonsils, was not significant $(P=0.167$ for Bray-Curtis, $P=0.607$ for unweighted UniFrac, and $P=0.281$ for weighted UniFrac) (Fig. 3). Next, we performed ANOSIM and PERMANOVA to identify the characteristics responsible for beta-diversity, and found that the total serum IgE levels were a significant factor (Supplementary Table 1). When the tonsils or adenoids were individually evaluated to explore possible correlations between 


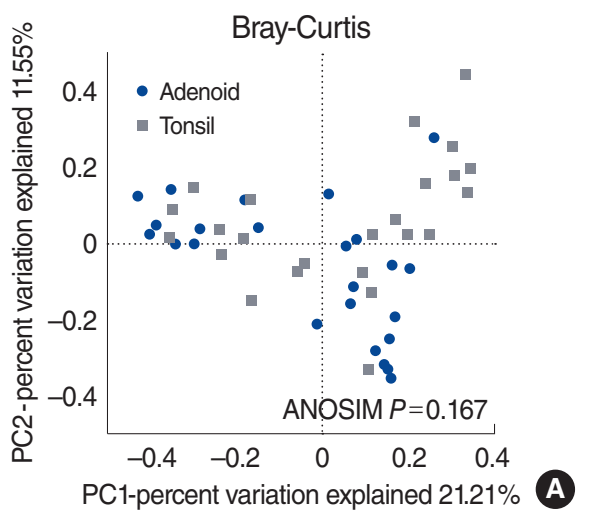

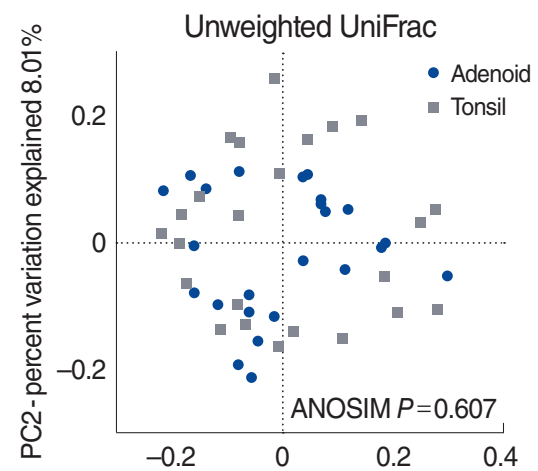

PC1-percent variation explained $12.22 \%$

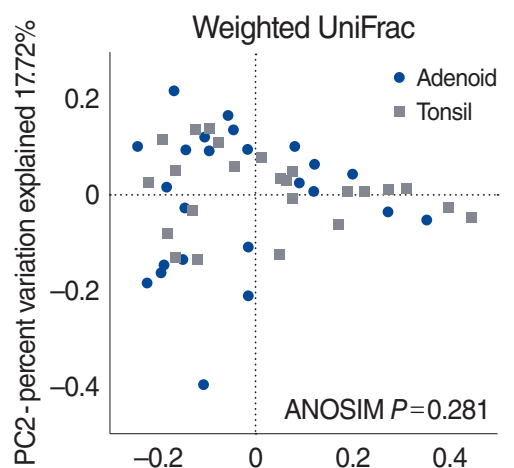

PC1-percent variation explained $42.54 \%$ C

Fig. 3. Beta-diversity (dissimilarity of intra-individual adenotonsillar microbiomes) in the adenoid and tonsil samples. Principal component analysis of Bray-Curtis (A), unweighted UniFrac (B), and weighted UniFrac (C) distances. Each point represents an individual's microbiota. ANOSIM, analysis of similarities.

Table 3. Correlations between beta-diversity indices and clinical characteristics

\begin{tabular}{lccc}
\hline & \multicolumn{3}{c}{ P-value } \\
\cline { 2 - 4 } Characteristics & $\begin{array}{c}\text { Weighted } \\
\text { UniFrac }\end{array}$ & $\begin{array}{c}\text { Unweighted } \\
\text { UniFrac }\end{array}$ & Bray-Curtis \\
\hline PERMANOVA & & & \\
Sex & 0.408 & 0.585 & 0.470 \\
Positivity of MAST & 0.185 & 0.174 & 0.186 \\
Distance analysis & & & \\
OSA-18 (sleep disturbance) & 0.663 & 0.813 & 0.714 \\
OSA-18 (physical suffering) & 0.750 & 0.284 & 0.296 \\
OSA-18 (emotional stress) & 0.265 & 0.079 & 0.852 \\
OSA-18 (daytime problems) & 0.855 & 0.845 & 0.463 \\
OSA-18 (caregiver concern) & 0.898 & 0.356 & 0.430 \\
Adenoid IL-8 (pg/mL) & 0.253 & 0.183 & 0.160 \\
Adenoid Hsp27 (pg/mL) & 0.386 & 0.083 & 0.267 \\
Adenoid Hsp70 (pg/mL) & 0.519 & 0.348 & 0.722 \\
Tonsil IL-8 (pg/mL) & 0.316 & 0.131 & 0.074 \\
Tonsil Hsp27 (pg/mL) & 0.545 & 0.226 & 0.038 \\
& & & $($ (rho $=-0.425)$ \\
Tonsil Hsp70 (pg/mL) & 0.293 & 0.849 & 0.288 \\
White blood cell (109 $/ \mathrm{L})$ & 0.134 & 0.001 & 0.037 \\
& & $($ rho $=0.610)$ & $($ rho $=0.426)$ \\
Eosinophil count (10 $/ \mathrm{L})$ & 0.817 & 0.779 & 0.792 \\
\hline
\end{tabular}

PERMANOVA, permutational multivariate analysis of variance; MAST, multiple allergen simultaneous test; OSA, obstructive sleep apnea; IL-8, interleukin-8; Hsp, heat shock protein.

beta-diversity and clinical characteristics, no significant factor was identified, with the exception of the total IgE level (data not shown). The relationship of clinical characteristics with the betadiversity of the entire adenotonsillar microbiome was further analyzed based on the average weighted UniFrac, unweighted UniFrac, and Bray-Curtis distances, which represented intraindividual dissimilarities in the adenotonsillar microbiota. The Hsp27 level in the tonsil LFs $(P=0.038$, rho $=0.288$ for Bray-Curtis) and WBC counts $(P=0.001$, rho $=0.610$ for unweighted UniFrac; $P=0.037$, rho $=0.426$ for Bray-Curtis) were responsible for beta-diversity (Table 3).

\section{DISCUSSION}

To the best of our knowledge, this is the first study to evaluate the associations of the adenotonsillar microbiome with clinical characteristics, including extracellular immune response-associated protein concentrations and subjective quality of life, in pediatric patients with snoring. Our findings supported the hypothesis that mucosal microbes and host mucosal immune responses exhibit mutual effects and correlate closely with each other.

Only two studies have evaluated the paired microbiome from the tonsils and adenoids in pediatric patients without infections. One study evaluated microbiome variation based on sampling sites (surface swab and tissue biopsy samples); however, it did not find different compositions between the tonsils and adenoids [4]. The other study suggested an overlap between the bacterial composition of the tonsils and adenoids, although they showed an overall dissimilarity [8]. Our study showed that the general microbiome composition was not meaningfully different between the adenoids and tonsils, although differences were found in several genera. Therefore, we consider our current comparative study to be inconclusive, and recommend further studies using a larger patient population.

We hypothesized that the microbiome of tonsils and adenoids might be related to the severity of subjective symptoms in pediatric patients with snoring. When comparing the tonsil or adenoid microbiome with clinical characteristics, we could not find any significant factors, except the total serum IgE level (data not shown). However, we found that a domain of the OSA-18 questionnaire (emotional stress) was negatively associated with the alpha-diversity of the adenotonsillar microbiome, suggesting that lower alpha-diversity of the microbiome was linked to higher levels of emotional stress. IL-8, which was correlated with al- 
pha-diversity in this study, has been reported to be present at higher levels in the pharyngeal LFs of patients with OSA than in healthy controls, and IL-8 levels were found to be associated with the apnea-hypopnea index in patients with OSA [20]. Reduced bacterial diversity affects disease pathogenesis and is associated with inflammatory conditions in the nasal cavity [21]. The clinical severity of Alzheimer disease is closely correlated with gut bacterial diversity [22]. Consistent with our finding that subjective discomfort was associated with regional microbiome diversity, mood disorders have also been associated with reduced diversity of the gut microbiome [23]. Although the mechanisms are unclear, our data suggest that microbiome diversity might be associated with the subjective severity and pathogenesis of snoring in pediatric patients.

The surface of adenoids and tonsils is composed of folds and crypts, which are colonized by microbiota. These microbes influence the development of the innate mucosal immune system, which plays an important role in protecting against mucosal infection $[24,25]$. Hsp27 and Hsp70 have been reported to be expressed in the pediatric adenoid tissues and occur in the extracellular areas, where they can function as immune mediators by regulating bacterial population [10]. The possibility that Hsp70 might play an important role in the pathogenesis of adenoid hyperplasia has also been suggested [26]. Therefore, we evaluated the correlations between the adenotonsillar microbiome and immunity-associated molecules on the mucosal surface. Beta-diversity analysis showed significant differences in the microbial composition of adenotonsillar tissues depending on the level of Hsp27 in the LF and WBC counts. In the cytoplasm, Hsp27 (a small Hsp) responds to stress and acts as a chaperone to facilitate appropriate protein folding. Outside the cell, Hsp27 is involved in modulating the inflammatory response by interacting with certain receptors [27]. Hsp27 is present in the adenoid tissue and LFs, and is associated with the microbiome [10]. Thus, our findings support the hypothesis that the regional microbial composition interacts with components of the host mucosal immunity, and we demonstrated this interaction at the mucosal surface of tonsils and adenoids.

We previously evaluated the association between AR and the nasal mucosal microbiome [13]. When we evaluated the adenotonsillar microbiome based on the presence of AR or total serum IgE levels, only the total serum IgE levels were found to be associated with dissimilarity in the intra-individual adenotonsillar microbiome (Supplementary Fig. 1). However, the presence of AR is considered to be correlated with adenotonsillar hypertrophy. For example, the presence of adenoid hypertrophy was demonstrated to increase the severity and duration of AR, suggesting a correlation between adenoid hypertrophy and AR [28]. Since AR is an important factor that affects the local regional microbiome, this issue requires further evaluation.

Our study had several limitations. First, we analyzed a relatively small cohort of pediatric patients with snoring, which should be taken into consideration while interpreting the findings. Second, we used biopsy samples, including the surface mucosa and submucosal areas. The sampling site is an important factor that determines the variability of the microbiome in the adenotonsillar area [25]. Therefore, a different method or sampling location could produce different findings. Third, although the regional microbiome diversity and dissimilarities were associated with surface immune molecules, this was an observational study, and further research to evaluate the presence of a causal relationship is warranted. Finally, since our participants did not undergo polysomnography, we could not evaluate correlations of the adenotonsillar microbiome with the objective degree of sleep apnea or oxygen concentration. The relationship between the adenotonsillar microbiome and severity of sleep apnea therefore needs to be evaluated in future studies.

Our study demonstrated that adenotonsillar microbial diversity and intra-individual dissimilarity were associated with mucosal surface immune molecules and subjective discomfort in pediatric patients with snoring. The findings suggest that the regional microbiome might functionally affect the subjective severity of snoring in pediatric patients and closely interact with components of the regional immune responses at the mucosal surface of the adenoids and tonsils in pediatric patients. This could have important implications for understanding the pathogenesis of pediatric snoring and OSA.

\section{CONFLICT OF INTEREST}

No potential conflict of interest relevant to this article was reported.

\section{ACKNOWLEDGMENTS}

This research was supported by a National Research Foundation of Korea (NRF) grant funded by the Korean government (MSIP) (NRF- 2017R1A1A1A05000760 to HJM).

\section{ORCID}

Kyung Soo Kim https://orcid.org/0000-0003-2637-0555

Hyun Jin Min https://orcid.org/0000-0003-3075-1350

\section{AUTHOR CONTRIBUTIONS}

Conceptualization: HJM. Data curation, Formal analysis: all authors. Funding acquisition: HJM. Methodology, Project administration, \& Visualization: all authors. Writing-original draft: HJM. Writing-review \& editing: KSK. 


\section{SUPPLEMENTARY MATERIALS}

Supplementary materials can be found via https://doi.org/10. 21053/ceo.2020.01634.

\section{REFERENCES}

1. Gerhardsson H, Stalfors J, Odhagen E, Sunnergren O. Pediatric adenoid surgery in Sweden 2004-2013: incidence, indications and concomitant surgical procedures. Int J Pediatr Otorhinolaryngol. 2016 Aug;87:61-6.

2. Juul ML, Rasmussen ER, Rasmussen SH, Sorensen CH, Howitz MF. A nationwide registry-based cohort study of incidence of tonsillectomy in Denmark, 1991-2012. Clin Otolaryngol. 2018 Feb;43(1): 274-84.

3. Zautner AE.Adenotonsillar disease. Recent Pat Inflamm Allergy Drug Discov. 2012 May;6(2):121-9.

4. Johnston J, Hoggard M, Biswas K, Astudillo-Garcia C, Radcliff FJ, Mahadevan M, et al. Paired analysis of the microbiota between surface tissue swabs and biopsies from pediatric patients undergoing adenotonsillectomy. Int J Pediatr Otorhinolaryngol. 2018 Oct;113: 51-7.

5. Xu Z,WuY,Tai J, Feng G, GeW, Zheng L, et al. Risk factors of obstructive sleep apnea syndrome in children. J Otolaryngol Head Neck Surg. 2020 Mar;49(1):11.

6. Johnston J, Hoggard M, Biswas K, Astudillo-Garcia C, Waldvogel-Thurlow S, Radcliff FJ, et al. The bacterial community and local lymphocyte response are markedly different in patients with recurrent tonsillitis compared to obstructive sleep apnoea. Int J Pediatr Otorhinolaryngol. 2018 Oct;113:281-8.

7. Tsaoussoglou M, Lianou L, Maragozidis P, Hatzinikolaou S, Mavromati M, Orologas N, et al. Cysteinyl leukotriene receptors in tonsillar B- and T-lymphocytes from children with obstructive sleep apnea. Sleep Med. 2012 Aug;13(7):879-85.

8. Fago-Olsen H, Dines LM, Sorensen $\mathrm{CH}$, Jensen A. The adenoids but not the palatine tonsils serve as a reservoir for bacteria associated with secretory otitis media in small children. mSystems. 2019 Feb; 4(1):e00169-18.

9. Maw AR. Chronic otitis media with effusion and adeno-tonsillectomy: a prospective randomized controlled study. Int J Pediatr Otorhinolaryngol. 1983 Dec;6(3):239-46.

10. Min HJ, Park JS, Kim CE, Kim KS. Profiling of heat shock proteins 27 and 70 in adenoids of children. Eur Arch Otorhinolaryngol. 2019 Sep;276(9):2483-9.

11. WHO Collaborating Center for Asthma and Rhinitis, Bousquet J, Anto JM, Demoly P, Schunemann HJ, Togias A, et al. Severe chronic allergic (and related) diseases: a uniform approach: a MeDALL-GA2LEN-ARIA position paper. Int Arch Allergy Immunol. 2012;158(3): 216-31.
12. Kim JK, Yoon YM, Jang WJ, Choi YJ, Hong SC, Cho JH. Comparison study between MAST CLA and OPTIGEN. Am J Rhinol Allergy. 2011 Jul-Aug;25(4):e156-9.

13. Hyun DW, Min HJ, Kim MS, Whon TW, Shin NR, Kim PS, et al. Dysbiosis of inferior turbinate microbiota is associated with high total IgE levels in patients with allergic rhinitis. Infect Immun. 2018 Mar; 86(4):e00934-17.

14. Ishman SL, Yang CJ, Cohen AP, Benke JR, Meinzen-Derr JK, Anderson RM, et al. Is the OSA-18 predictive of obstructive sleep apnea: comparison to polysomnography. Laryngoscope. 2015 Jun;125(6): 1491-5.

15. Hamady M, Walker JJ, Harris JK, Gold NJ, Knight R. Error-correcting barcoded primers for pyrosequencing hundreds of samples in multiplex. Nat Methods. 2008 Mar;5(3):235-7.

16. Caporaso JG, Kuczynski J, Stombaugh J, Bittinger K, Bushman FD, Costello EK, et al. QIIME allows analysis of high-throughput community sequencing data. Nat Methods. 2010 May;7(5):335-6.

17. Kim J, Bhattacharjee R, Dayyat E, Snow AB, Kheirandish-Gozal L, Goldman JL, et al. Increased cellular proliferation and inflammatory cytokines in tonsils derived from children with obstructive sleep apnea. Pediatr Res. 2009 Oct;66(4):423-8.

18. Segata N, Izard J, Waldron L, Gevers D, Miropolsky L, Garrett WS, et al. Metagenomic biomarker discovery and explanation. Genome Biol. 2011 Jun;12(6):R60.

19. Wang X, Li H, Bezemer TM, Hao Z. Drivers of bacterial beta diversity in two temperate forests. Ecol Res. 2016 Jan;31:57-64.

20. Vicente E, Marin JM, Carrizo SJ, Osuna CS, Gonzalez R, Marin-Oto $\mathrm{M}$, et al. Upper airway and systemic inflammation in obstructive sleep apnoea. Eur Respir J. 2016 Oct;48(4):1108-17.

21. De Boeck I, Wittouck S, Martens K, Claes J, Jorissen M, Steelant B, et al.Anterior nares diversity and pathobionts represent sinus microbiome in chronic rhinosinusitis. mSphere. 2019 Nov;4(6):e00532-19.

22. Liu P,Wu L, Peng G, Han Y,Tang R, Ge J, et al. Altered microbiomes distinguish Alzheimer's disease from amnestic mild cognitive impairment and health in a Chinese cohort. Brain Behav Immun. 2019 Aug; 80:633-43.

23. Jiang H, Ling Z, Zhang Y, Mao H, Ma Z, Yin Y, et al. Altered fecal microbiota composition in patients with major depressive disorder. Brain Behav Immun. 2015 Aug;48:186-94.

24. Perry M,Whyte A. Immunology of the tonsils. Immunol Today. 1998 Sep;19(9):414-21.

25. Johnston JJ, Douglas R.Adenotonsillar microbiome: an update. Postgrad Med J. 2018 Jul;94(1113):398-403.

26. Aktepe F, Sahin O, Dilek H, Yilmaz D, Kahveci O, Derekoy S. Immunohistochemical assesment of heat shock protein 70 in adenoid tissue. Int J Pediatr Otorhinolaryngol. 2007 Jun;71(6):857-61.

27. Reddy VS, Madala SK, Trinath J, Reddy GB. Extracellular small heat shock proteins: exosomal biogenesis and function. Cell Stress Chaperones. 2018 May;23(3):441-54.

28. Dogru M, Evcimik MF, Calim OF. Does adenoid hypertrophy affect disease severity in children with allergic rhinitis? Eur Arch Otorhinolaryngol. 2017 Jan;274(1):209-213. 